\title{
Adherencia a la suplementación con vitamina $D$ y factores determinantes de ella, durante el primer año de vida
}

\author{
MÓNICA ARANCIBIA C. ${ }^{1}$, MARÍA LORETO REYES G. ${ }^{2}$, JAIME CERDA L. ${ }^{3}$ \\ 1. Residente de Pediatría, División de Pediatría, Pontificia Universidad Católica de Chile. \\ 2. Endocrinóloga Infantil. División de Pediatría. Escuela de Medicina. Pontificia Universidad Católica de Chile. \\ 3. Pediatra. Departamento de Salud Pública. Escuela de Medicina. Pontificia Universidad Católica de Chile.
}

\section{ABSTRACT \\ Adherence to vitamin D supplementation and determinant factors during the first year of life}

Introduction: Breastfed infants under one year of age may not get enough vitamin $\mathrm{D}$; therefore a vitamin supplement is needed. The adherence to this policy has not yet been evaluated in Chile. Objective: To evaluate the adherence to vitamin D supplementation in children less than one year old and the determinant factors involved. Patients and Method: A cross-sectional study was carried out in three Catholic University Health Network centers. Breastfed infants under one year of age were included in the study. Their parents/guardians filled out a questionnaire about adherence to supplementation and its determinant factors. Results: 170 infants were recruited. 164 of them received supplementation, with a good adherence of $68.9 \%$. The main reason for non-adherence was due to maternal forgetfulness. The identified risk factor for poor adherence was the number of maternal children. Conclusions: Vitamin D supplementation in Chile reaches high levels, but its adherence is poor. More education to parents on ways to avoid forgetting the supplement is needed as well as on identifying risk factors during medical consultations.

(Key words: Supplementation, vitamin D, adherence).

Rev Chil Pediatr 2014; 85 (4): 428-436

\section{RESUMEN}

Introducción: Los lactantes menores de un año alimentados con leche materna poseen múltiples factores que impiden una adecuada adquisición de vitamina $\mathrm{D}$, haciendo la suplementación necesaria. La adherencia a esta política de salud no ha sido evaluada en Chile. Objetivo: Evaluar la adherencia a la suplementación con vitamina $\mathrm{D}$ en niños menores de un año y conocer los factores determinantes que intervienen en ella. Pacientes y Método: Estudio transversal en tres Centros de Salud pertenecientes a Red de Salud Universidad Católica.

Recibido el 03 de abril de 2013, última versión aceptada para publicación el 07 de abril de 2014.

Potenciales conflictos de interés: Este trabajo cumple con los requisitos sobre consentimiento /asentimiento informado, comité de ética, financiamiento, estudios animales y sobre la ausencia de conflictos de intereses según corresponda. 
Se reclutaron lactantes menores de 1 año que recibían lactancia materna y se realizó una encuesta a sus tutores indagando sobre adherencia a la suplementación y factores determinantes de ésta. Resultados: Se reclutaron 170 lactantes. Recibían suplementación 164 de ellos, reportando buena adherencia en un 68,9\%. La principal causa para no adherir fue el olvido materno. El factor de riesgo identificado para mala adherencia fue el número de hijos maternos. Conclusiones: La suplementación con vitamina D en Chile alcanza niveles altos, pero la adherencia a ésta es deficiente. Se necesita de mayor educación a los padres al respecto, reforzando maneras de evitar el olvido e identificando los factores de riesgo en todas las consultas médicas.

(Palabras clave: Suplementación, vitamina D, adherencia).

Rev Chil Pediatr 2014; 85 (4): 428-436

\section{Introducción}

La suplementación de vitamina $\mathrm{D}$ se ha demostrado eficiente y necesaria para la prevención del raquitismo en lactantes alimentados con leche materna. En el presente se ha mostrado que la vitamina $D$ puede tener efectos beneficiosos más allá del metabolismo fosfo-cálcico. Muchos de sus efectos son debidos a que es una hormona esteroidal capaz de modular la programación temprana de la salud humana a largo plazo. El mecanismo por el cual realiza esto es aún desconocido, pero se cree que podría estar relacionado con el fenómeno de impresión metabólica (metabolic imprinting) que se produce durante los períodos críticos del desarrollo humano ${ }^{1}$. Su efectos positivos estarían en de diversos sistemas biológicos, destacando: a) homeostasis del calcio y del metabolismo óseo; b) función cardíaca y regulación de la presión arterial; c) sistema inmune adaptativo e innato; d) algunas glándulas endocrinas (hipófisis, paratiroides, páncreas, suprarrenal, tiroides, ovarios, testículos y corteza adrenal); e) sistema locomotor y f) cerebro ${ }^{2-5}$. Además, se describe que ayudaría a prevenir algunos tipos de cáncer (colon, próstata, mama y cerebro) y la aparición de enfermedades autoinmunes (psoriasis, diabetes tipo 1, enfermedad inflamatoria intestinal y esclerosis múltiple) $)^{1,2,4,6,7}$. Por todo lo anteriormente señalado, garantizar un aporte óptimo de vitamina $\mathrm{D}$ durante toda la vida es de fundamental importancia para favorecer el buen funcionamiento de múltiples órganos, sobre todo durante los períodos más críticos del desarrollo (feto, neonato, lactante).

La academia americana de pediatría (AAP) el 2003 recomendó que todo niño mayor de 1 mes debía recibir 200 unidades internacionales (UI) de vitamina D para cubrir los requerimientos básicos. Esto se modificó el 2008, cuando la nueva recomendación de la AAP fue publicada señalando que todo niño desde los primeros días de vida debía recibir 400 UI de vitamina $\mathrm{D}$ al día ${ }^{8}$.

El organismo cuenta con dos formas para obtener vitamina $\mathrm{D}$, la absorbe desde los alimentos o la sintetiza en la piel al exponerla a la luz solar (rayos ultravioleta) ${ }^{4}$. Los factores que alteran estos procesos y que por consiguiente producen déficit de vitamina $\mathrm{D}$ son variados $\mathrm{y}$ cambian según la edad ${ }^{6}$.

Un grupo etario importante de revisar son los recién nacidos y lactantes menores de un año, ya que poseen múltiples factores que impiden una adecuada adquisición de vitamina $\mathrm{D}$ tanto del ambiente como endógena: la lactancia materna tiene bajo contenido de vitamina $\mathrm{D}$, fórmulas de inicio y de continuación sólo si se consume más de 1 litro diario cumple con los requerimientos recomendados por AAP, en menores de 6 meses no se recomienda la exposición solar, en mayores de 6 meses se usan protectores solares y no consumen cantidad suficiente de alimentos ricos en vitamina D. De lo anterior se desprende que en este grupo etario la suplementación es necesaria por lo que debe indicarse en todo recién nacido y lactante.

Existen algunos estudios que muestran la adherencia de los pediatras a indicar la suplementación con vitamina $\mathrm{D}$ en niños menores de 1 año alimentados sólo con leche materna. Davenport M et al, el 2004 realizó encuestas en Carolina del Norte que mostraron que el 
$44,6 \%$ de ellos recomendaba la suplementación ${ }^{9}$, similar a lo reportado por Shaikh U et al, el 2004 en un estudio en Las Vegas en que el $48 \%$ de los pediatras la indicaban ${ }^{10}$. El 2009 se indagó sobre la conducta de los pediatras que trabajan en el Servicio Militar de Estados Unidos mostrando que el $89 \%$ de ellos recomendaban el uso de vitamina $\mathrm{D}^{11}$ y el 2010 se publicó que el $36,4 \%$ de un grupo de pediatras de Seattle lo hacía ${ }^{12}$.

Sobre la adherencia de los padres a seguir esta recomendación hay escasos datos en la literatura. El 2010 se publicaron en USA dos estudios, uno de ellos reveló una baja adherencia a la suplementación con vitamina $\mathrm{D}$ en lactantes menores alimentados tanto con leche materna exclusiva, $10,5 \%$ como con lactancia mixta, 8,5\%, recibiendo al menos tres días a la semana suplementación ${ }^{8}$, y el otro determinó que la minoría de lactantes menores alimentados con lactancia materna recibía suplementación de forma rutinaria 15,9\%. Este último además encontró que la administración de la vitamina $\mathrm{D}$ se asociaba significativamente a dos factores: que los padres estuvieran de acuerdo en la suplementación indicada por el pediatra y la creencia familiar que la leche materna tenía todos los elementos nutricionales necesarios por lo que no necesita vitaminas ${ }^{12}$. El 2011 Pludowski $P$ et al, realizó un estudio en Polonia que mostraba que el $82,1 \%$ de los niños de 6 meses y el $60,2 \%$ de los niños de 12 meses recibía todos los días suplementación con 400 UI de vitamina $\mathrm{D}^{13}$. Halicioglu O et al, el 2012 realizó un estudio en Turquía que mostró que el $79 \%$ de niños de 4 meses alimentados con leche materna recibían suplementación diaria ${ }^{14}$.

Actualmente no hay literatura que señale qué ocurre con la adherencia en países latinoamericanos, ni que indague de forma extensa sobre los factores determinantes de ésta que serían fundamentales para poder planificar estrategias sanitarias que ayuden a mejorarla.

El objetivo de este trabajo es conocer la adherencia a la suplementación con vitamina $\mathrm{D}$ en niños menores de 1 año y en forma secundaria saber la adherencia de los profesionales de salud a seguir la recomendación de la AAP $\mathrm{y}$ evaluar los factores determinantes que inter- vienen en la adherencia de la suplementación con vitamina $\mathrm{D}$.

\section{Pacientes y Método}

Estudio de tipo transversal de sección de corte. Se reclutaron lactantes menores de 1 año que realizaban sus controles sanos en tres diferentes Centros de Salud de la Red Universidad Católica (UC) en la especialidad de Pediatría de la Región Metropolitana. La muestra fue obtenida por conveniencia de forma consecutiva entre febrero y noviembre del año 2012. Se consideraron criterios de inclusión lactantes menores de 1 año que recibían lactancia materna y cuyos padres accedían a firmar consentimiento informado y de exclusión aquellos lactantes menores de 1 año que al momento de nacer fueron catalogados como prematuros y/o bajo peso nacimiento y aquellos que al momento de la encuesta fueran alimentados sólo con fórmulas de inicio o continuación o lactancia mixta con aporte de 400 UI de vitamina D por fórmula (mayor a 1 litro diario).

Los tutores de los lactantes que cumplían los criterios de selección fueron invitados a participar en el estudio mientras se encontraban en la sala de espera. A los que aceptaban se les solicitaba el consentimiento informado y posteriormente el investigador principal realizaba una encuesta. Al finalizar se contestaban dudas y se hacía educación sobre la suplementación con vitamina $\mathrm{D}$ a aquellos que lo solicitaban y se ofrecía a los tutores que tenían una mala indicación o adherencia.

La encuesta realizada incluía: identificación general del tutor y del menor, alimentación recibida por el lactante, características de la suplementación con vitamina D (indicación médica, frecuencia, dosis), factores determinantes de adherencia (causas de no adherencia, factores socioeconómicos, factores relación médico paciente y morbilidad familiar) (Anexo 1).

Se generó una base de datos en Microsoft ${ }^{\circledR}$ Excel 2010, que fue procesada con el programa SPSS. Se calculó la adherencia a la suplementación con vitamina D considerando la administración durante el mes previo a la en- 
cuesta. Se categorizó como buena adherencia aquellos que recibían suplementación el 90\% del tiempo o más (al menos 27 días de los 30 días previos) y mala adherencia quienes la recibían menos del 90\% del tiempo. Esta variable también fue analizada según el número exacto de días durante el último mes en que se administró la suplementación. Los factores determinantes de la adherencia estudiados mediante análisis univariado fueron: tipo de alimentación del lactante, ingreso promedio familiar, educación materna, edad del niño, edad materna, presencia de enfermedad crónica materna, antecedente o presencia actual de depresión materna, número de hijos. Esto se analizó primero tomando a la muestra en su totalidad y luego separándola en dos grupos: lactantes de 1 mes a 5 meses de vida y lactantes de 6 a 11 meses de edad. Las variables categóricas fueron analizadas mediante Chi cuadrado y las variables continuas mediante Mann-Whitney. El estudio fue aprobado por el Comité de ética de la Pontificia Universidad Católica de Chile y el consentimiento informado fue otorgado por sus padres.

\section{Resultados}

Se reclutó a un total de 170 personas, de quienes un $100 \%$ aceptó contestar la encuesta. De éstos $164(96,4 \%)$ recibían suplementación con vitamina D y 144 (88\%) de ellos tenía indicación médica adecuada para cumplir las 400 UI recomendadas. El 100\% de aquellos que no recibían suplementación eran mayores de 6 meses y no lo hacían porque su pediatra había suspendido la suplementación a pesar de que con la alimentación no alcanzaban a cumplir las 400 UI de vitamina D (figura 1).

De los 164 niños que si recibían suplementación, $106(64,6 \%)$ tenían entre 1 a 5 meses de edad y $58(35,4 \%)$ entre 6 y 11 meses. Las características de estos niños se detallan en la tabla 1.

En relación a la adherencia a la suplementación con vitamina D, 113 niños $(68,9 \%)$ fueron clasificados con buena adherencia, (tabla 2). Si se desglosa el cumplimiento de la administración de vitamina $\mathrm{D}$ por días durante el último mes, el 37,8\% de los niños recibía suplementación de forma diaria y todos recibían al menos la mitad del tiempo (3 veces por semana o más), (tabla 3). De los 102 niños que no recibían todos los días la vitamina $\mathrm{D}, 80$ de ellos $(78,4 \%)$ fue por olvido materno, $9(8,8 \%)$ por vómitos del lactante, $7(6,8 \%)$ porque la madre no compró el medicamento, 4 (3,9\%) por enfermedad del lactante y $2(1,9 \%)$ por otro motivo.

El estudio univariado realizado sobre los posibles factores determinantes de la adheren-

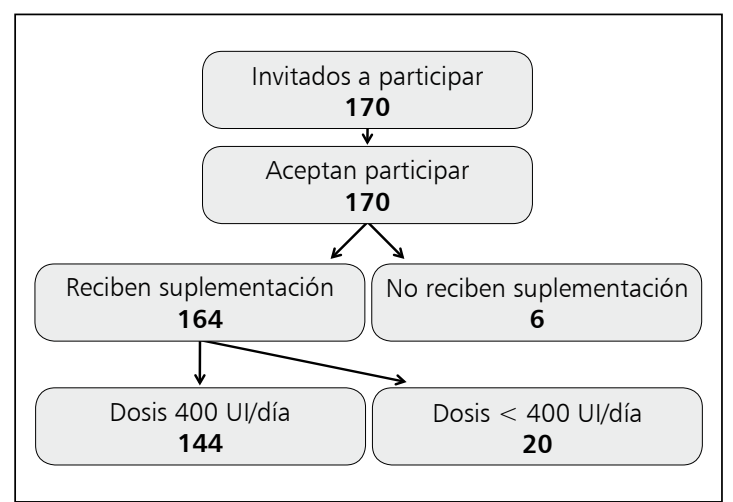

Figura 1. Participantes del estudio e indicación médica de suplementación con vitamina D.

Tabla 1. Características de los niños suplementados con vitamina D

\begin{tabular}{|c|c|}
\hline \multicolumn{2}{|l|}{ Variable $(n=164)$} \\
\hline \multicolumn{2}{|l|}{ Sexo } \\
\hline Femenino $(n, \%)$ & $70(42,7)$ \\
\hline Masculino (n, \%) & $97(55,3)$ \\
\hline Edad meses (promedio, DS) & $4,7(2,8)$ \\
\hline \multicolumn{2}{|l|}{ Alimentación láctea } \\
\hline Lactancia materna (n, \%) & $115(70,1)$ \\
\hline Lactancia mixta (n, \%) & $40(29,9)$ \\
\hline \multicolumn{2}{|l|}{ Tipo de familia } \\
\hline Monoparental (n, \%) & $29(17,7)$ \\
\hline Biparental (n, \%) & $135(82,3)$ \\
\hline \multicolumn{2}{|l|}{ Ingreso promedio familiar } \\
\hline$<\$ 300.000(\mathrm{n}, \%)$ & $30(18,3)$ \\
\hline$\$ 300.000$ a $\$ 700-000(n, \%)$ & $54(32,9)$ \\
\hline$>\$ 700.000(n, \%)$ & $80(48,8)$ \\
\hline \multicolumn{2}{|l|}{ Educación materna } \\
\hline Básica (n, \%) & $4 \quad(2,4)$ \\
\hline Media (n, \%) & $40(24,4)$ \\
\hline Superior (n, \%) & $120(73,2)$ \\
\hline
\end{tabular}

n: número, \%: porcentaje, DS: desviación estándar. 
Tabla 2. Adherencia a suplementación con vitamina $D$ según categorías

\begin{tabular}{|c|c|c|}
\hline Adherencia & n $(\%)$ & IC $95 \%$ \\
\hline $\begin{array}{l}\text { Buena (mayor al } 90 \% \text { de los días } \\
\text { durante el último mes) }\end{array}$ & $113(68,9)$ & $61,3-75,4$ \\
\hline $\begin{array}{l}\text { Mala (menor al } 90 \% \text { de los días } \\
\text { durante el último mes) }\end{array}$ & $51 \quad(31,1)$ & $24,4-38,5$ \\
\hline
\end{tabular}

Tabla 3. Adherencia a suplementación con vitamina $D$ según número de días

\begin{tabular}{|ccc|}
\hline n días/mes & $\mathbf{n}$ & $\mathbf{( \% )}$ \\
30 días & 62 & $(37,8)$ \\
29 días & 13 & $(7,9)$ \\
\hline 28 días & 23 & $(14,0)$ \\
27 días & 15 & $(9,1)$ \\
\hline 26 días & 10 & $(6,1)$ \\
25 días & 17 & $(10,4)$ \\
24 días & 3 & $(1,8)$ \\
23 días & 4 & $(2,4)$ \\
22 días & 2 & $(1,2)$ \\
20 días & 6 & $(3,7)$ \\
16 días & 2 & $(1,2)$ \\
15 días & 7 & $(4,3)$ \\
\hline
\end{tabular}

n: número, \%: porcentaje.

cia mostró que sólo el número de hijos presentaba una tendencia lineal estadísticamente significativa $(\mathrm{p}<0,02)$ aumentando el riesgo de mala adherencia al aumentar el número de hijos. Aquellos que tenían un hijo presentaban una buena adherencia en un $76,1 \%$ con un Odds ratio de 1,0; los que tenían dos hijos adherían bien en un $63,3 \%$ con un Odds ratio de 1,85 (IC 95\% 0,90-3,79) y por último los que tenían 3 o más hijos presentaban buena adherencia en un $50 \%$ con un Odds ratio de 3,19 (IC 95\% 1,07 a 9,54).

\section{Discusión}

Nuestro estudio muestra que la mayoría de los lactantes recibía suplementación con vitamina $\mathrm{D}$, pero que la adherencia a ésta es un problema en la población que consulta en la
Red de Salud UC al igual que en otros países. La adherencia de los profesionales de salud para recomendar la suplementación fue de un 96,4\%; indicando la dosis de $400 \mathrm{UI} / \mathrm{d}$ en un $88 \%$ de los lactantes. Si bien ambas cifras son elevadas lo que demuestra un conocimiento adecuado de las últimas recomendaciones de la AAP, cabe destacar que un $15,5 \%$ de los profesionales de salud realiza una indicación de suplementación inadecuada.

Al analizar la adherencia de los padres a la suplementación vemos que sólo un $37,8 \%$ de los niños la recibía de forma diaria, porcentaje menor que el reportado en la literatura ${ }^{13,14}$. Esto es importante si consideramos que los niveles plasmáticos de vitamina D (25 hidroxi-vitamina D) descritos en la población pediátrica para definir deficiencia de vitamina $\mathrm{D}$ son $<20$ ng/ml ${ }^{15,16}$ y Halicioglu et al, el 2012 demostró que niños de 4 meses que recibían suplementación diaria lograban niveles de $30,1 \pm 10,9$ ng/dl y aquellos que recibía entre 6 a 3 veces por semana niveles de $16,8 \pm 7,9 \mathrm{ng} / \mathrm{dl}^{14}$. De lo anterior se desprende que si existe pobre adherencia probablemente el riesgo de tener deficiencia de vitamina $\mathrm{D}$ es mayor, aunque esto requiere un estudio adicional en Chile.

Dentro de las causas de mala adherencia descritas la más frecuente fue el olvido materno, diferente a lo publicado por Taylor et al, el 2010 en Seattle, donde la causa principal fue la creencia de los padres que la leche materna tenía todos los nutrientes necesarios ${ }^{12}$ y por Gallo et al, el 2010 en Montreal donde el factor más relevantes fue la creencia materna de que al iniciar alimentación por fórmula ya no se requería suplementación ${ }^{17}$. Esta diferencia podría explicarse por el nivel educacional de las madres de la población estudiada y porque actualmente la información sobre la leche materna y vitamina D son más asequibles. Por lo tanto, para mejorar la adherencia podría reforzarse las técnicas para evitar el olvido como crear una rutina diaria al momento de administrarlas. Las demás causas reportadas podrían manejarse preguntando dirigidamente si existe algún problema al momento de la administración o alguna duda sobre la indicación para reforzar la idea de que siempre debe administrarse. Otra línea de investigación podría evaluar 
la eficacia, seguridad y adherencia del uso de vitamina D semanal o mensual.

En relación a las variables determinantes de la adherencia estudiadas, la única relevante fue el número de hijos de la madre. Dado esto, se puede sugerir hacer mayor hincapié sobre el propósito, los beneficios y la importancia del cumplimiento de la suplementación con vitamina $\mathrm{D}$ en los controles médicos a las madres con más de un hijo, sobre todo con más de dos.

Dentro de las limitaciones que tiene nuestro estudio cabe mencionar que se usó muestra de conveniencia, que corresponde a un nivel educacional y socioeconómico no representativo de la población chilena. Además, nuestra encuesta no fue validada y las respuestas a ella eran dependientes de la memoria de tutor.

\section{Agradecimientos}

Agradecemos a las familias que participaron en estudio, así como a los docentes que contribuyeron al buen desarrollo y presentación.

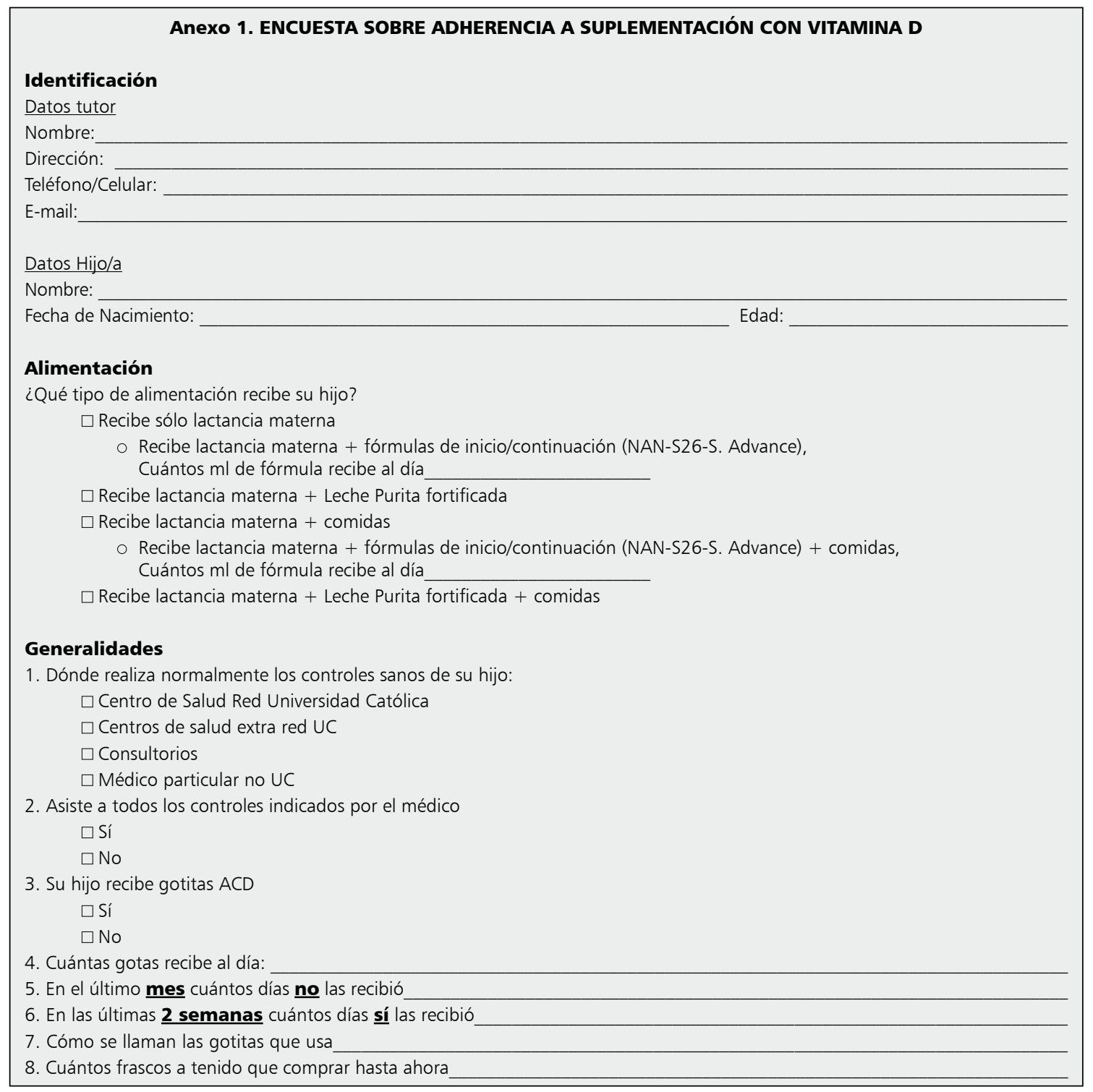


9. Administración de las gotas

$\square$ Pone las gotas en una cuchara

$\square$ Se las da directamente

$\square$ Otra:

\section{Factores determinantes de adherencia}

1. Si su hijo no recibe diariamente sus gotas, la explicación sería

$\square$ Dificultades en la administración:

$\square$ No le gustan las gotas al niño

$\square$ Vomita o regurgita las gotas

$\square$ Otra:

$\square$ Olvido

$\square$ Se me acabaron y no he comprado aún

$\square$ No son necesaria ya que la lactancia materna tiene todos los nutrientes $\square$ Otra:

\section{Factores socioeconómicos}

1. Comuna de residencia:

2. Edad materna y paterna (años)

Madre:

Padre:

3. Nivel de educación materno y paterno (hasta que curso estudió)

Madre:

Padre:

4. Profesión:

Madre:

Actualmente ejerce:

$\square$ Sí $\square$ No

Su ocupación actual es:

Tiene contrato laboral: $\quad \square$ Sí $\quad \square$ No

Considera su trabajo estable: $\square$ Sí $\quad \square$ No

Cuanto tiempo lleva trabajando en el mismo lugar: Padre:
Actualmente ejerce:
$\square$ Sí $\quad \square$ No
Su ocupación actual es:
Tiene contrato laboral:

$\square$ Sí $\square$ No
Considera su trabajo estable: $\square$ Sí $\quad \square$ No
Cuanto tiempo lleva trabajando en el mismo lugar:

5. Estado marital y constitución familiar
$\square$ Casada
$\square$ En pareja
$\square$ Soltera
$\square$ Viuda
$\square$ Divorciada

a. $N^{\circ}$ de hijos

b. Orden de este hijo $\left(1^{\circ}, 2^{\circ}\right.$, etc $)$

6 . Red de apoyo social

a. Con quién vive:

b. Quién ayuda en la crianza y cuidado del niño (abuelos, tíos, jardín, etc.)

c. Existe alguien con quien pueda hablar de todo
$\square$ Sí
$\square$ No

7. El sustento económico de la familia del menor lo aporta
$\square$ El padre
$\square$ La madre
$\square$ Padre y madre
$\square$ Abuelos
Otro: 
8. El ingreso promedio de cada mes es:

$\square$ Menos de 100.000

$\square$ Entre 100.000 a 300.000

$\square$ Entre 300.000 a 500.000

$\square$ Entre 500.000 a 700.000

$\square$ Más de 700.000

9. Actualmente está cursando dificultades económicas

$\square$ Sí

$\square$ No

\section{Factores relación médico-paciente}

1. ¿Generalmente asiste a controles con el mismo profesional? ¿Por qué?

$$
\square \text { Sí: }
$$

$\square$ No:

2. Con respeto a las indicaciones que le recomienda su médico, usted diría que:

$\square$ Siempre confía en lo indicado

$\square$ Generalmente confía en lo indicado

$\square$ Pocas veces confía en lo indicado

$\square$ Casi nunca confía en lo indicado

$\square$ Otra:

3. Con respeto a las indicaciones que le recomienda su médico, usted diría que:

$\square$ Siempre sigue las indicaciones

$\square$ Generalmente sigue las indicaciones

$\square$ Pocas veces sigue las indicaciones

$\square$ Casi nunca sigue las indicaciones

$\square$ Otra:

4. Usted diría que siente confianza para preguntarle sus dudas al médico:
$\square$ Siempre
$\square$ Generalmente
$\square$ Pocas veces
$\square$ Casi nunca
$\square$ Otra:

5. ¿Existe una buena acogida del médico a las preguntas?

$$
\begin{aligned}
& \square \text { Sí } \\
& \square \text { No }
\end{aligned}
$$

6. ¿Se siente satisfecha con la explicación sobre la necesidad del uso de las gotitas ACD?

$$
\begin{aligned}
& \square \text { Sí } \\
& \square \text { No }
\end{aligned}
$$

\section{Morbilidad familiar}

1. Hay antecedente de enfermedad crónica de la madre (diabetes, hipertensión, etc.)

$$
\begin{aligned}
& \square \text { Sí } \\
& \square \text { No }
\end{aligned}
$$

2. Si la respuesta anterior fue positiva, usted diría que:

$\square$ Diariamente se toma los medicamentos indicados, sin olvidarse ningún día

$\square$ Generalmente se toma los medicamentos indicados, sólo se olvida 1 a 2 veces por semana

$\square$ Sólo le toma los medicamentos cuando se enferma o no se los toma porque cree que ya no los necesita

3. Hay antecedentes de enfermedad psiquiátrica materna:

$$
\begin{aligned}
& \square \text { Otra: } \\
& \square \text { No }
\end{aligned}
$$$$
\square \text { Depresión }
$$

4. Hay antecedente de algún hijo con patología crónica (hermano del lactante)

$$
\begin{aligned}
& \square \text { Sí } \\
& \square \text { No }
\end{aligned}
$$

5. Si la respuesta anterior fue positiva, usted diría que:

$\square$ Diariamente le da los medicamentos indicados, sin olvidarse ningún día

$\square$ Generalmente le da los medicamentos indicados, sólo se olvida 1 a 2 veces por semana

$\square$ Sólo le da los medicamentos cuando se enferma o no se los da porque cree que ya no los necesita

MUCHAS GRACIAS!!!!! 


\section{Referencias}

1.- Kaludjerovic J, Vieth R: Relationship Between Vitamin D During Perinatal Development and Health. J Midwifery \& Women's Health 2010; 55: 550-60.

2.- Norman A: From vitamin D to hormone D: fundamentals of the vitamin D endocrine system essential for good health. Am J Clin Nutr 2008; 88 (suppl): 491S-9S.

3.- Nemere I, Farach-Carson MC: Membrane receptors for steroid hormones: a case for specific cell surface binding sites for vitamin D metabolits and estrogens. Biochem Biophys Res Commun 1998; 248: 442-9.

4.- Prentice A, Goldberg G, Schoenmakers I: Vitamin D across the lifecycle: physiology and biomarkers. Am J Clin Nutr 2008; 88 (suppl): 500S-6.

5.- Walker V, Modlin R: The Vitamin D Connection to Pediatric Infections and Immune function. Pediatr Res 2009; 65 (5 Pt 2): 106R-13.

6.- Peterlik M, Boonen S, Cross H, Lamberg-Allardt C: Vitamin D and Calcium Insufficiency-Related Chronic Diseases: an Emerging World-Wide Public Health Problem. Int. J. Environ. Res Public Health 2009; 6: 2585-607.

7.- Holick M: Vitamin D: importance in the prevention of cancers, type 1 diabetes, heart disease, and osteoporosis. Am J Clin Nutr 2004; 79: 362-71.

8.- Perrine C, Sharma A, Jefferds $M$, Serdula M, Scanlon $K$ : Adherence to Vitamin D Recommendations Among US Infants. Pediatrics 2010; 125: 627-32.

9.- Davenport M, Uckun A, Calikoglu A: Pediatrician patterns of prescribing vitamin supplementation for infants: do they contribute to rickets? Pediatrics 2004; 113 (1 pt 1): 179-80.

10.- Shaikh U, Alpert P: Practices of vitamin D recommendation in Las Vegas, Nevada. J Hum Lact 2004; 20 (1): 56-61.

11.- Sherman E, Svec R: Barriers to vitamin D supplementation among military physicians. Mil Med 2009; 174 (3): 302-7.

12.- Taylor J, Geyer L, Feldman K: Use of Supplemental Vitamin D Among Infants Breastfed for Prolonged Periods. Pediatrics 2010; 125: 105-11.

13.- Pludowski P, Socha P, Karczmarewicz E, et al: Vitamin D Supplementation and Status in Infants: A Prospective Cohort Observational Study JPGN 2011; 53: 93-9.

14.- Halicioglu O, Sutcuoglu S, Koc F, Yildiz O, Akman $S$, Aksit $S$ : Vitamin D Status of Exclusively Breastfed 4-Month-Old Infants Supplemented During Different Seasons. Pediatrics 2012; 130: e921-7.

15.- Munns C, Zacharin M, Rodda $C$, et al: Paediatric Endocrine Group; Paediatric Bone Australasia. Prevention and treatment of infant and childhood vitamin D deficiency in Australia and New Zealand: a consensus statement. Med J Aust 2006; 185 (5): 268-72.

16.- Institute of Medicine. Dietary Reference Intakes for Calcium and Vitamin D. Washington, DC: The National Academies Press; 2011. http://www.ncbi.nlm.nih.gov/ books/NBK56070/

17.- Gallo S, Jean-Philippe S, Rodd C, Weiler H: Vitamin D supplementation of Canadian Infants: practices of Montreal mothers. Applied Physiology, Nutrition \& Metabolism 2010; 35 Issue 3: p303-9. 6p. 